\title{
Behavioral Analysis of Practitioners Who Frequently Perform Facial Thread-Lifting Procedures
}

\author{
Seung Min Oh, MD, MMBA ${ }^{1}$ \\ Bong Cheol Kim, $\mathrm{MD}^{2}$ \\ Min Seung Kim, $M^{3}$ \\ Sang Bong Lee, $\mathrm{MD}^{4}$ \\ Woo Kyung Bae, MD, MPH \\ ${ }^{1}$ ON Clinic, Seoul, Korea \\ 2Lamar Clinic Isu Branch, Seoul, Korea \\ ${ }^{3}$ Inee Clinic, Seoul, Korea \\ ${ }^{4}$ Pygmalion Clinic, Seoul, Korea \\ ${ }^{5}$ Health Promotion Center, Seoul National \\ University Bundang Hospital, Seongnam, Korea
}

Received October 14, 2021

Accepted October 28, 2021

\section{Correspondence}

Woo Kyung Bae

Health Promotion Center, Seoul National University Bundang Hospital, Seongnam 13620, Korea

E-mail:65597@snubh.org

https://orcid.org/0000-0001-6729-4128

(C) Korean Society for Laser, Dermatology and Trichology

(a) This is an open access article distributed under the terms of the Creative Commons Attribution NonCommercial License (http://creativecommons.org/ licenses/by-nc/4.0) which permits unrestricted noncommercial use, distribution, and reproduction in any medium, provided the original work is properly cited.

\section{Background and Objectives}

The effects of thread-lifting remain controversial because of the lack of a standardized protocol. To examine the treatment practices of Korean doctors who frequently perform thread-lifting.

\section{Materials and Methods}

At a large-scale aesthetic conference in Korea, we conducted a survey including clinicians who practice facial thread-lifting and analyzed the procedures and preferences of the doctors who participated in the questionnairebased survey. We then compared the treatment characteristics of clinicians who perform thread-lifting at least once per week (high-frequency) to those of clinicians who perform it less than once per week (low-frequency) using multivariate regression analyses.

\section{Results}

Overall, 243 clinicians participated in the questionnaire-based survey, and the responses of 150 participants who currently perform thread-lifting were analyzed. The number of threads injected per hemi-face was higher in the high-frequency group than in the low-frequency group. Furthermore, the high-frequency group was more likely to use molding cog threads than the low-frequency group.

\section{Conclusion}

Practitioners who frequently perform thread-lifting are likely to prefer molding cog threads and insert a relatively large number of threads per face. These results facilitate the development of a standard thread-lifting procedure and standard evaluation protocol for the effectiveness of this procedure.

\section{Key words}

Thread lifting, Protocol of thread lifting, Molding thread, Cog thread 


\section{INTRODUCTION}

Thread-lifting is a procedure that is widely performed for facial rejuvenation and has a large market [1,2]. However, the lifting effect of the procedure remains controversial. In 2018-2019, systematic reviews of the effects of threadlifting were published [3-5]. In one of these reviews, seven existing studies conducted using four types of thread were analyzed; however, the types of thread and techniques used differed, and the effects of thread-lifting were subjectively evaluated. The authors concluded that there were no specific effects, excluding the results of two studies that were conducted under the sponsorship of a particular company [3].

Despite the uncertainty about its effects, the threadlifting procedure is still being actively performed. Recently, several additional studies have been published showing the effectiveness of thread-lifting [6-10]. Therefore, no consensus has been reached on the value of this procedure.

Tissue experiments and comparative clinical trials have been performed to examine the lifting effect of this procedure; however, each doctor has different treatment methods, and these studies are not standardized. Among the studies included in the systematic review by Haydar et al., there were no split face studies or patient control studies, and only one blinded surgeon-scoring study was included [3].

Therefore, a standard protocol and further studies are needed to delineate the lifting effect of the procedure. A standard protocol should be designed based on the lifting principle used and demonstrated safety. We aimed to study

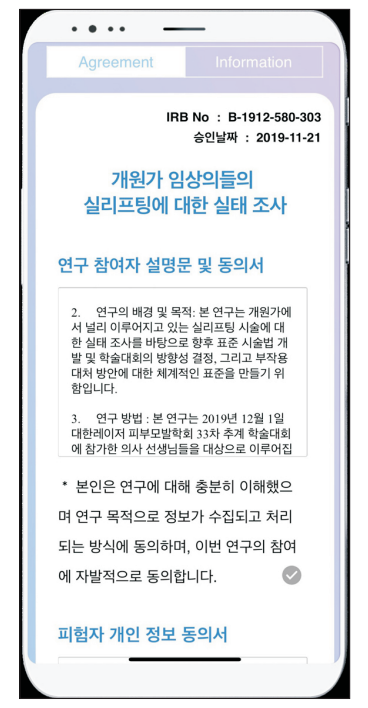

Fig. 1. IRB related content on the first screen of web based questionnaire. the characteristics of practitioners who frequently perform thread-lifting through the use of a questionnaire to help standardize this procedure.

\section{MATERIALS AND METHODS}

\section{Participants and survey design}

A survey was conducted for clinicians who attended the $33^{\text {rd }}$ fall Korean Association for Laser, Dermatology, and Trichology conference, held in Seoul, Republic of Korea, on December 1, 2019. The survey was conducted in a webbased, real-time, mobile environment. The researchers sent a text message with a link to the survey to attendees who registered at the conference. Attendees could participate in the survey by clicking the link in the text message. Each link contained a unique encrypted number, allowing it to be accessed only once, thus preventing duplicate questionnaire participation. Therefore, even if the link was forwarded to another person's cell phone, the survey could not be resubmitted. By clicking the link, those who participated in the web survey started the questionnaire after reading the IRBrelated content on the first screen and consenting to participate in the questionnaire (Fig. 1). After entering their gender and age, each participant answered a survey consisting of 13 multiple-choice questions (Fig. 2). These questions investigated the frequency of treatment, experience with the procedure, preferred products, preferred techniques, and side effects.

\section{Statistics}

The participants were divided into two groups: those who performed the procedure one or more times per week

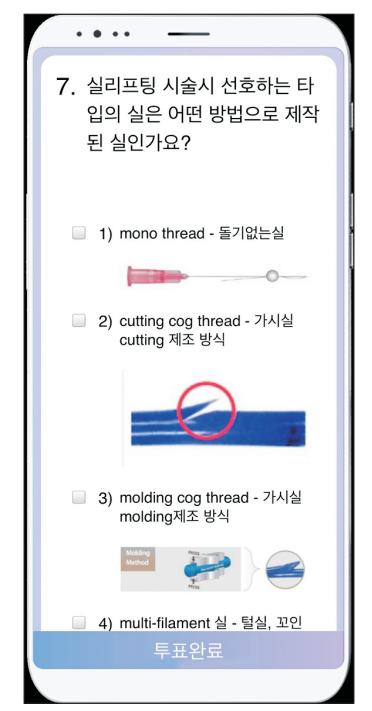

Fig. 2. Web survey with 13 multiple-choice questions. 
and those who performed the procedure less than once per week. The participant's age was analyzed using a t-test. Gender, professional/operational experience, education method, technique, and the number of threads injected per hemi-face were analyzed using the Chi-square test. Multiple responses were given for questions regarding thread type, preferred product, and preferred technique, and each item was analyzed using the Chi-square test. An ordered regression analysis was performed on the number of threads injected per hemi-face according to the frequency of treatment using age, gender, specialized subjects, career experience, and education method as disturbance variables. Logistic regression was performed for thread type, preferred product, and preferred technique. Stata version 15.0 (StataCorp. 2017. Stata Statistical Software: Release 15. College Station, TX: StataCorp LLC.) was used for statistical analysis of the survey results.

\section{Ethics}

Ethics approval was obtained from the Institutional Review Board of Seoul National University Bundang Hospital (B-1912/580-303).

\section{RESULTS}

\section{Baseline demographics}

Among the clinicians who participated in the academic conference, 243 completed the questionnaire. Of these, 150 were analyzed, excluding 93 , who answered that they never performed the thread-lifting procedure.

The demographic and sociological characteristics of the survey participants are shown in Table 1 . The participants were divided into two groups, depending on how frequently they performed the thread-lifting procedure: those that performed the procedure less than once a week (lowfrequency) and those that performed the procedure one or more times a week (high-frequency).

There were no differences in gender or preferred education method between the two groups. The high-frequency group had higher percentages of aesthetic physicians ( $48.3 \%$ vs. $36.7 \%)$ and plastic surgeons ( $13.3 \%$ vs. $4.4 \%)$ than the low-frequency group. Moreover, the percentage of participants who had more than one year of experience was higher in the high-frequency group than in the low-frequency group (51/60, $85.0 \%$ vs. $57 / 90,63.3 \%)$.

\section{Univariate analysis}

Univariate analysis was performed on the procedure characteristics between the two groups (Table 2). Regarding

Table 1. The demographic and sociological characteristics of the participants

\begin{tabular}{|c|c|c|c|c|}
\hline Characteristics & Total $(n=150)$ & $<1 /$ week $(n=90)$ & $>1 /$ week $(n=60)$ & p-value \\
\hline Age (years) & $42.5 \pm 8.8$ & $42.9 \pm 8.9$ & $41.8 \pm 8.7$ & \\
\hline Specialty & & & & 0.050 \\
\hline Aesthetic physician & $62(41.3)$ & $33(36.7)$ & $29(48.3)$ & \\
\hline Dermatologist & $18(12.0)$ & $12(13.3)$ & $6(10.0)$ & \\
\hline Plastic surgeon & $12(8.0)$ & $4(4.4)$ & $8(13.3)$ & \\
\hline Other & $58(38.7)$ & $41(45.6)$ & $17(28.3)$ & \\
\hline Experience & & & & 0.008 \\
\hline$<1$ month & $7(4.7)$ & $6(6.7)$ & $1(1.7)$ & \\
\hline 1 month < 1 year & $35(23.3)$ & $27(30.0)$ & $8(13.3)$ & \\
\hline 1 year $<3$ years & $34(22.7)$ & $15(16.7)$ & $19(31.7)$ & \\
\hline 3 years $<5$ years & $30(20.0)$ & $21(23.3)$ & $9(15.0)$ & \\
\hline$>5$ years & $44(29.3)$ & $21(23.3)$ & $23(38.3)$ & \\
\hline Education & & & & 0.903 \\
\hline Self-education & $15(10.0)$ & $10(11.1)$ & $5(8.3)$ & \\
\hline Small seminar & $67(44.7)$ & $42(46.7)$ & $25(41.7)$ & \\
\hline Hands-on education & $45(30.0)$ & $26(28.9)$ & $19(31.7)$ & \\
\hline Standard training & $5(3.3)$ & $2(2.2)$ & $3(5.0)$ & \\
\hline Large conference & $16(10.7)$ & $9(10.0)$ & $7(11.7)$ & \\
\hline Other & $2(1.3)$ & $1(1.1)$ & $1(1.7)$ & \\
\hline
\end{tabular}

Numbers are presented as mean \pm standard deviation or number (\%). 


\section{The \\ AESTHETICS}

the type of thread most frequently used for the procedure, 95 (63.3\%) survey participants use molding cog thread , 59 (39.9\%) use cutting cog thread, and $47,(31.3 \%)$ use mono thread. Regarding preferred products, 73 (48.7\%) survey participants prefer $\mathrm{N}-\operatorname{cog}^{\circledast}$ ( $\mathrm{N}$-finders Co. Ltd), 71 (47.3\%) prefer Mint ${ }^{\circledR}$ (Hans Biomed Co. Ltd), and 56 (37.3\%) prefer Blue Rose Forte ${ }^{\circledast}$ (Hugel Co. Ltd). No significant differences were observed between groups in the type of thread used or preferred products.

When asked how many threads are injected per hemi-

Table 2. Characteristics of practice according to the procedure frequency

\begin{tabular}{|c|c|c|c|c|}
\hline Factor & Total $(n=150)$ & $<1 /$ week $(n=90)$ & $\geq 1 /$ week $(n=60)$ & p-value \\
\hline \multicolumn{5}{|c|}{ Thread type (multiple response) } \\
\hline Mono-thread & 47 (31.3) & $28(31.1)$ & $19(31.7)$ & 0.943 \\
\hline Cutting cog & $59(39.9)$ & $35(38.9)$ & $24(40.0)$ & 0.891 \\
\hline Molding cog & $95(63.3)$ & $52(57.8)$ & $43(71.7)$ & 0.084 \\
\hline Multi-filament & $20(13.3)$ & $14(15.6)$ & $6(10.0)$ & 0.327 \\
\hline Mesh & $17(11.3)$ & $12(13.3)$ & $5(8.3)$ & 0.344 \\
\hline Cone type & $34(22.7)$ & $21(23.3)$ & $13(21.7)$ & 0.811 \\
\hline Others & $6(4.0)$ & $4(4.4)$ & $2(3.3)$ & 0.734 \\
\hline Number of threads & & & & 0.092 \\
\hline 1-5/hemi-face & $87(58.7)$ & $59(65.6)$ & $29(48.4)$ & \\
\hline 6-10/hemi-face & $53(35.3)$ & $26(28.9)$ & $27(45.0)$ & \\
\hline$>$ 11/hemi-face & $9(6.0)$ & $5(5.6)$ & $4(6.7)$ & \\
\hline \multicolumn{5}{|c|}{ Preferred technique (multiple response) } \\
\hline Mono thread & $58(38.7)$ & $32(35.6)$ & $26(43.3)$ & 0.338 \\
\hline Bidirectional cog & $91(60.7)$ & $53(58.9)$ & $38(63.3)$ & 0.585 \\
\hline Multi-directional cog & $77(51.3)$ & $49(54.4)$ & $28(46.7)$ & 0.350 \\
\hline Multi-filament & $21(14.0)$ & $12(13.3)$ & $9(15.0)$ & 0.773 \\
\hline Mesh type thread & $15(10.0)$ & $9(10.0)$ & $6(10.0)$ & 1.000 \\
\hline Fascia fixation & $34(22.7)$ & $19(21.1)$ & $15(25.0)$ & 0.577 \\
\hline Others & $10(6.6)$ & $6(6.6)$ & $4(6.7)$ & 0.353 \\
\hline
\end{tabular}

Numbers are presented as number (\%).

Thread type, preferred product, and preferred technique items are multiple responses.
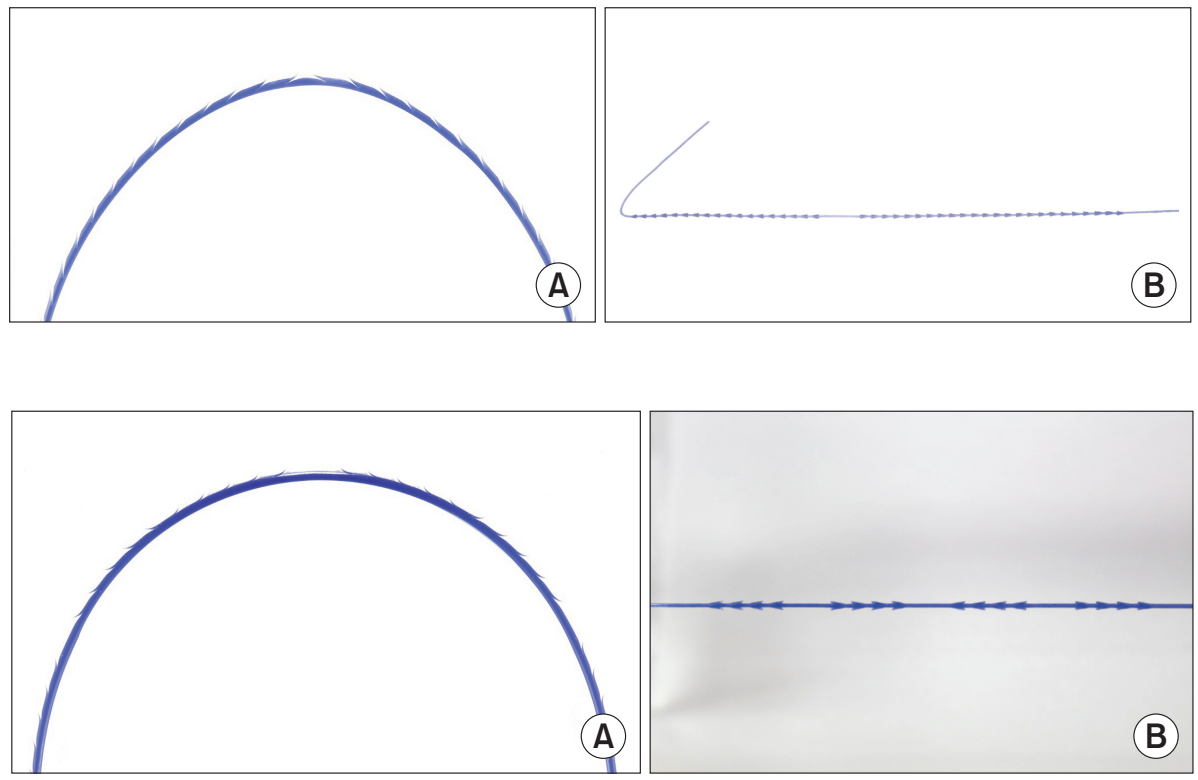

Fig. 3. (A) $\mathrm{N}-\operatorname{cog}^{\circledR}$ thread, (B) Blue Rose Forte ${ }^{\circledR}$ thread. 
Table 3. Multivariate analysis of treatment preferences according to procedure frequency

\begin{tabular}{|c|c|c|c|c|}
\hline Factors & Variables & OR & $95 \%$ confidence interval & p-value \\
\hline Number of threads & & $2.901 *$ & $1.299-6.478$ & 0.009 \\
\hline \multirow[t]{6}{*}{ Thread type (multiple response) } & Mono-thread & $1.729 * *$ & $0.726-4.118$ & 0.217 \\
\hline & Cutting cog thread & $0.895 * *$ & $0.413-1.943$ & 0.780 \\
\hline & Molding cog thread & $2.353 * *$ & $1.006-5.502$ & 0.048 \\
\hline & Multi-filament thread & $0.616 * *$ & $0.185-2.043$ & 0.428 \\
\hline & Mesh thread & $0.487 * *$ & $0.143-1.658$ & 0.250 \\
\hline & Cone type thread & $0.493 * *$ & 0.168-1.441 & 0.196 \\
\hline \multirow[t]{6}{*}{ Preferred technique (multiple response) } & Mono thread & $1.502 * *$ & $0.686-3.288$ & 0.309 \\
\hline & Bidirectional cog & $0.987 * *$ & $0.455-2.139$ & 0.973 \\
\hline & Multi-directional cog & $0.787 * *$ & $0.362-1.710$ & 0.545 \\
\hline & Multi-filament & $2.066 * *$ & $0.617-6.911$ & 0.239 \\
\hline & Mesh type & $1.004 * *$ & $0.293-3.440$ & 0.995 \\
\hline & Fascia fixation & $1.315 * *$ & $0.540-3.203$ & 0.547 \\
\hline
\end{tabular}

In each analysis, age, gender, specialty, career, or preferred education method was used as the disturbance variable.

*OR was calculated using ordered regression analysis.

**OR was calculated using logistic regression analysis.

face, 87 (58.0\%) participants in the survey answered 3-5, $53(35.3 \%)$ responded $6-10$, and $9(6.0 \%)$ responded 11 or more. Regarding preferred technique, 91 (60.7\%) survey participants prefer bi-directional cog thread insertion (Fig. 3A, N-cog ${ }^{\oplus}$; Fig. 3B, Blue Rose Forte $\left.{ }^{\circledR}\right), 77$ (51.3\%) prefer multi-directional cog thread insertion (Fig. 4A, N-cog ${ }^{\oplus}$; Fig. $\left.4 B, \mathrm{QTL}^{\circledR}\right)$, and 58 (38.7\%) prefer mono thread insertion. No significant difference in preferred technique was observed between groups.

\section{Multivariate analysis}

Multivariate analysis was performed between the two frequency groups (Table 3). The high-frequency group used more threads per hemi-face than the low-frequency group (odds ratio $[O R], 2.901 ; p=0.009$ ). Furthermore, molding cog thread was more likely to be used by the highfrequency group compared to other threads (OR, 2.353; $p=$ 0.048 ). There were no statistically significant differences in preferred products or techniques.

\section{DISCUSSION}

To the best of our knowledge, this is the first study to analyze the demographic and sociological characteristics of clinicians who practice the thread-lifting procedure. The high-frequency group injected more threads per face than the low-frequency group. In addition, molding cog threads were used more frequently in the high-frequency group compared to the low-frequency group.

The physical traction of the cog causes the thread-lifting effect at the beginning of the procedure and chemical ef- fects, including neocollagenesis, that continuously occur in the tissue afterward [11]. Through experiments in pigs, Yoon et al. [11] observed the histological changes that occurred when the thread was injected into the body over one year, and systematically summarized these changes. They demonstrated that the lifting and tightening effects were produced by the neocollagenesis and fibrous bridging phenomena occurring in the surrounding tissue after thread injection. Furthermore, Lee et al. [12] revealed in animal experiments that the degree of neocollagenesis that appears after thread insertion is independent of the type of material and is proportional to the area of the surface area of the material. Based on the results of these studies, inserting a larger number of cog threads would create stronger traction and increase neocollagenesis. We speculate that the doctors who perform frequent procedures might have observed that inserting a relatively large number of threads leads to better sustainability and satisfying results of threadlifting. Analyzing the frequency of practitioners' procedures may be used as a reference in making standard procedures.

Although the effectiveness of thread-lifting has not been demonstrated by meta-analyses [3-5], these analyses were based on studies with various treatment methods that have not proved to be effective. Analyzing the procedures of practitioners who frequently perform thread-lifting may facilitate the development of standard procedures. In the absence of a structured training curriculum and standard procedure for thread-lifting, clinicians are sharing their treatment methods through seminars, live procedure training, and experience. A standardized procedure based on our understanding of the principles of thread-lifting is need- 
ed; only then can the effects of thread-lifting be accurately evaluated.

One limitation of this study was that the differences between the two groups were analyzed in the absence of evidence that those in the high-frequency group had better outcomes than those in the low-frequency group. However, our results suggest a starting point for future research based on the analysis of the problems of previous results. For example, the information that high-frequency practitioners insert a large number of threads is useful for practitioners to develop effective procedures and perform various studies.

Our data was self-reported and is subject to the inherent biases of such data collection. Further studies should be designed to collect prospective, concrete procedure characteristics and related outcomes, predefined in detail derived from the standardization of the thread-lifting procedure.

In the future, research on the principles and safety of the procedure according to various factors, such as the type of thread, protocol, number of threads, and design method, should be conducted. These results would facilitate the development of a standardized thread-lifting procedure and allow an accurate re-evaluation of the effects of threadlifting.

\section{ACKNOWLEDGEMENTS}

We give special thanks to the Chairman of KALDAT, Hyung Moon Kim, and other members for permitting this research at their conference. We also thank Beom Jin Shin, the CEO of MADEINREAL Co., LTD, and Dong Hyun Kim, the Development Manager of MADEINREAL Co., LTD, who developed the web-based survey program and implemented it on the day of the survey.

\section{CONFLICT OF INTEREST}

No potential conflict of interest relevant to this article was reported.

\section{REFERENCES}

1. Villa MT, White LE, Alam M, Yoo SS, Walton RL. Barbed sutures: a review of the literature. Plast Reconstr Surg 2008; 121:102e-8e.

2. Garvey PB, Ricciardelli EJ, Gampper T. Outcomes in threadlift for facial rejuvenation. Ann Plast Surg 2009;62:482-5.

3. Gülbitti HA, Colebunders B, Pirayesh A, Bertossi D, van der Lei B. Thread-lift sutures: still in the lift? A systematic review of the literature. Plast Reconstr Surg 2018;141:341e-7e.

4. Obourn CA, Williams EF 3rd. A decade of thread-lifting-what have we learned over the last 10 years? JAMA Facial Plast Surg 2018;20:349-50.

5. Tong LX, Rieder EA. Thread-lifts: a double-edged suture? A comprehensive review of the literature. Dermatol Surg 2019; 45:931-40.

6. Lee $\mathrm{H}$, Yoon $\mathrm{K}$, Lee $\mathrm{M}$. Outcome of facial rejuvenation with polydioxanone thread for Asians. J Cosmet Laser Ther 2018; 20:189-92.

7. Ogilvie MP, Few JW Jr, Tomur SS, Teven CM, Semersky AJ, Bruno CR, et al. Rejuvenating the face: an analysis of $100 \mathrm{ab}$ sorbable suture suspension patients. Aesthet Surg J 2018;38: 654-63.

8. Wattanakrai K, Chiemchaisri N, Wattanakrai P. Mesh suspension thread for facial rejuvenation. Aesthetic Plast Surg 2020; 44:766-74.

9. Ali YH. Two years' outcome of thread lifting with absorbable barbed PDO threads: innovative score for objective and subjective assessment. J Cosmet Laser Ther 2018;20:41-9.

10. Suh DH, Jang HW, Lee SJ, Lee WS, Ryu HJ. Outcomes of polydioxanone knotless thread lifting for facial rejuvenation. Dermatol Surg 2015;41:720-5.

11. Yoon JH, Kim SS, Oh SM, Kim BC, Jung W. Tissue changes over time after polydioxanone thread insertion: an animal study with pigs. J Cosmet Dermatol 2019;18:885-91.

12. Lee CG, Jung J, Hwang S, Park CO, Hwang S, Jo M, et al. Histological evaluation of bioresorbable threads in rats. Korean J Clin Lab Sci 2018;50:217-24. 